Bull. Korean Math. Soc. 48 (2011), No. 5, pp. 1003-1014

http://dx.doi.org/10.4134/BKMS.2011.48.5.1003

\title{
CONVOLUTIONS OF WHITE NOISE OPERATORS
}

\author{
Un Cig Ji And Young Yi Kim
}

\begin{abstract}
Motivated by the convolution product of white noise functionals, we introduce a new notion of convolution products of white noise operators. Then we study several interesting relations between the convolution products and the quantum generalized Fourier-Mehler transforms, and study a quantum-classical correspondence.
\end{abstract}

\section{Introduction}

The white noise theory initiated by Hida [5] to give rigorous meaning of white noise as the time derivative of the Brownian motion has been extensively developed with wide applications to stochastic calculus, mathematical finance and mathematical physics, etc. One of the important applications of the white noise theory is to infinite dimensional analysis, specially infinite dimensional harmonic analysis, in which the convolution product [11] and Fourier-Gauss transforms [3, 11], generally with operator parameters [2], of white noise functionals were studied. The quantum white noise theory [8] has been developed based on the quantum decomposition of the (classical) white noise into the sum of the pointwise annihilation and creation operators. The quantum white noise theory plays an important role in the study of problems including singularities concerned with (white noise) operators, and then widely applied to quantum stochastic calculus $[6,10,12,15]$, see also [13] and references cited therein. On the other hand, a convolution of white noise operators was studied in [1] and the Fourier-Gauss transforms were generalized in [7] as transforms acting on white noise operators.

In this paper, motivated by the convolution product on white noise functionals and studies in [1], we introduce a new notion of convolution products of white noise operators and study several interesting relations between the convolutions and the quantum generalized Fourier-Mehler transforms of white noise operators.

Received February 24, 2010; Revised July 29, 2010.

2010 Mathematics Subject Classification. Primary 60H40; Secondary 46F25.

Key words and phrases. white noise operator, Wick product, convolution, quantum generalized Fourier-Mehler transform.

This work was supported by the research grant of the CBNU in 2009 . 
This paper is organized as follows: In Section 2 we recall basic notions and well-known results in white noise theory, which are necessary for our study, see $[11,13]$. In Section 3 we introduce convolution products of white noise operators and then study relations between the convolution products and the quantum generalized Fourier-Mehler transforms, finally we study a quantumclassical correspondence including various relations between (classical) convolution products and generalized Fourier-Mehler transforms.

\section{Preliminaries}

\subsection{Ridging of Fock space}

Let $H$ be a (complex) Hilbert space with norm $|\cdot|_{0}$. Let $A$ be a selfadjoint operator in $H$ such that $\left\|A^{-1}\right\|_{\mathrm{OP}}<1$ and $\left\|A^{-1}\right\|_{\mathrm{HS}}^{2}<\infty$. By the standard construction from $H$ and $A$, we have a Gelfand triple:

$$
E \subset H \subset E^{*},
$$

see $[11,14]$, where $E^{*}$ is the strong dual space of $E$. For each $p \in \mathbb{R},|\xi|_{p} \equiv$ $\left|A^{p} \xi\right|_{0}$. The topology of $E$ is defined by the Hilbertian norms $\left\{|\cdot|_{p}: p \in \mathbb{R}\right\}$, and then $E$ becomes a countable Hilbert nuclear space. The canonical bilinear form on $E^{*} \times E$ is denoted by $\langle\cdot, \cdot\rangle$.

The (Boson) Fock space over $H$, denoted by $\Gamma(H)$, is the Hilbert space consisting of all sequences $\left(f_{n}\right)_{n=0}^{\infty}$ such that $f_{n} \in H^{\widehat{\otimes} n}$ the $n$-fold symmetric tensor product of $H$ and $\sum_{n=0}^{\infty} n !\left|f_{n}\right|_{0}^{2}<\infty$, where $H^{\widehat{\otimes} 0}=\mathbb{C}$. Let $\Gamma(A)$ be the second quantization of the operator $A$ defined by

$$
\Gamma(A) \phi=\left(A^{\otimes n} f_{n}\right)_{n=0}^{\infty}, \quad \phi=\left(f_{n}\right)_{n=0}^{\infty} \in \Gamma(H) .
$$

Then $\Gamma(A)$ is a selfadjoint operator in $\Gamma(H)$ with $\left\|\Gamma(A)^{-1}\right\|_{\text {oP }}<1$ and $\left\|\Gamma(A)^{-1}\right\|_{\mathrm{HS}}<\infty$. By the standard construction from $\Gamma(H)$ and $\Gamma(A)$, we have a Gelfand triple:

$$
(E) \subset \Gamma(H) \subset(E)^{*},
$$

where $(E)$ is the space of all $\phi=\left(f_{n}\right)_{n=0}^{\infty}$ in $\Gamma(H)$ with $f_{n} \in E^{\widehat{\otimes} n}$ such that $\|\phi\|_{p}^{2}=\sum_{n=0}^{\infty} n !\left|f_{n}\right|_{p}^{2}<\infty$ for all $p \geq 0$ and $(E)^{*}$ is the strong dual space of $(E)$. Moreover, it is known that for each $\Phi \in(E)^{*}$ there exists a unique sequence $\left\{F_{n}\right\}_{n=0}^{\infty}$ with $F_{n} \in\left(E^{\otimes n}\right)_{\text {sym }}^{*}$ such that

$$
\langle\langle\Phi, \phi\rangle\rangle=\sum_{n=0}^{\infty} n !\left\langle F_{n}, f_{n}\right\rangle, \quad \phi=\left(f_{n}\right)_{n=0}^{\infty} \in(E) .
$$

For each $\xi \in E$, an exponential vector (or coherent vector) $\phi_{\xi}$ is defined by

$$
\phi_{\xi}=\left(1, \xi, \frac{\xi^{\otimes 2}}{2 !}, \ldots, \frac{\xi^{\otimes n}}{n !}, \ldots\right) .
$$

It is well-known that $\left\{\phi_{\xi}: \xi \in E\right\}$ spans a dense subspace of $(E)$. 


\subsection{White noise operators}

A continuous linear operator $\Xi$ from $(E)$ into $(E)^{*}$ is called a white noise operator. Let $\mathcal{L}\left((E),(E)^{*}\right)$ denote the space of all white noise operators equipped with the topology of bounded convergence. The symbol of $\Xi \in \mathcal{L}\left((E),(E)^{*}\right)$ is defined by

$$
\widehat{\Xi}(\xi, \eta)=\left\langle\left\langle\Xi \phi_{\xi}, \phi_{\eta}\right\rangle\right\rangle, \quad \xi, \eta \in E .
$$

Since $\left\{\phi_{\xi}: \xi \in E\right\}$ spans a dense subspace of $(E)$, every $\Xi \in \mathcal{L}\left((E),(E)^{*}\right)$ is uniquely determined by its symbol by virtue of the following theorem.

Theorem 2.1 ([9, 14]). Let $\Theta: E \times E \rightarrow \mathbb{C}$ be a $\mathbb{C}$-valued function. Then $\Theta$ is the symbol of an operator $\Xi \in \mathcal{L}\left((E),(E)^{*}\right)$ if and only if $\Theta$ is Gâteaux-entire and there exist constants $p \geq 0$ and $C, K \geq 0$ such that

$$
|\Theta(\xi, \eta)| \leq C e^{K\left(|\xi|_{p}^{2}+|\eta|_{p}^{2}\right)}, \quad \xi, \eta \in E .
$$

Moreover, $\Xi \in \mathcal{L}((E),(E))$ if and only if for any $p \geq 0$ and $\epsilon \geq 0$, there exist constants $C \geq 0$ and $q \geq 0$ such that

$$
|\Theta(\xi, \eta)| \leq C e^{\epsilon\left(|\xi|_{p+q}^{2}+|\eta|_{-p}^{2}\right)}, \quad \xi, \eta \in E .
$$

By applying Theorem 2.1, for each given $\Xi_{1}, \Xi_{2} \in \mathcal{L}\left((E),(E)^{*}\right)$, the Wick product $\Xi_{1} \diamond \Xi_{2} \in \mathcal{L}\left((E),(E)^{*}\right)$ of $\Xi_{1}$ and $\Xi_{2}$ is well-defined by

$$
\widehat{\Xi_{1} \diamond \Xi_{2}}(\xi, \eta)=\widehat{\Xi}_{1}(\xi, \eta) \widehat{\Xi}_{2}(\xi, \eta) e^{-\langle\xi, \eta\rangle}, \quad \xi, \eta \in E,
$$

see [4].

The symbol of the Gross Laplacian $\Delta_{\mathrm{G}} \in \mathcal{L}((E),(E))$ is given by

$$
\widehat{\Delta_{G}}(\xi, \eta)=\langle\xi, \xi\rangle e^{\langle\xi, \eta\rangle}, \quad \xi, \eta \in E
$$

see $[3,11,14]$.

On the other hand, for each $\Xi \in \mathcal{L}\left((E),(E)^{*}\right)$ we define $T$-symbol by

$$
T_{\mathrm{op}}(\Xi)(\xi, \eta)=\widehat{\Xi}(i \xi, i \eta) e^{-\frac{1}{2}(\langle\xi, \xi\rangle+\langle\eta, \eta\rangle)}, \quad \xi, \eta \in E,
$$

which is motivated by the T-transform of white noise functionals (see Section $4)$.

\section{Convolutions and transforms of white noise operators}

In this section, we introduce a new notion of convolution products of white noise operators and study some relations between the convolutions and the quantum generalized Fourier-Mehler transforms. 


\subsection{Convolution products of white noise operators}

Motivated by the convolution of white noise functionals (see Section 4 ), for each fixed $\Upsilon \in \mathcal{L}\left((E),(E)^{*}\right)$, we define a convolution $\Xi_{1} * \Upsilon \Xi_{2} \in \mathcal{L}\left((E),(E)^{*}\right)$ of two white noise operators $\Xi_{1}, \Xi_{2} \in \mathcal{L}\left((E),(E)^{*}\right)$ by

$$
\Xi_{1} * \Upsilon \Xi_{2}=\Xi_{1} \diamond \Xi_{2} \diamond \Upsilon \text {. }
$$

Here by applying Theorem 2.1 we see that $\Xi_{1} *_{\Upsilon} \Xi_{2}$ becomes a white noise operator.

Proposition 3.1. Let $\Upsilon \in \mathcal{L}\left((E),(E)^{*}\right)$ be given. Then for any $\Xi_{1}, \Xi_{2} \in$ $\mathcal{L}\left((E),(E)^{*}\right)$,

$$
T_{\mathrm{op}}\left(\Xi_{1} * \Upsilon \Xi_{2}\right)=T_{\mathrm{op}}\left(\Xi_{1}\right) T_{\mathrm{op}}\left(\Xi_{2}\right)
$$

if and only if $\Upsilon=e^{\frac{1}{2} \Delta_{G}^{*}} \Gamma(2 I) e^{\frac{1}{2} \Delta_{\mathrm{G}}}$.

Proof. By (3) and (1) we obtain that for any $\xi, \eta \in E$,

$$
\begin{aligned}
& T_{\mathrm{op}}\left(\Xi_{1} * \Upsilon \Xi_{2}\right)(\xi, \eta) \\
= & \left(\Xi_{1} \diamond \Xi_{2} \diamond \Upsilon\right)-(i \xi, i \eta) C(\xi, \eta) \\
= & \widehat{\Xi}_{1}(i \xi, i \eta) \widehat{\Xi}_{2}(i \xi, i \eta) \widehat{\Upsilon}(i \xi, i \eta) C(\xi, \eta) e^{2\langle\xi, \eta\rangle} \\
= & T_{\mathrm{op}}\left(\Xi_{1}\right)(\xi, \eta) T_{\mathrm{op}}\left(\Xi_{2}\right)(\xi, \eta) \widehat{\Upsilon}(i \xi, i \eta) C(i \xi, i \eta) e^{2\langle\xi, \eta\rangle},
\end{aligned}
$$

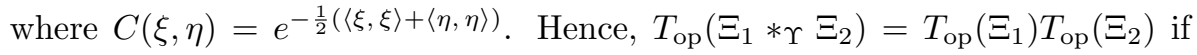
and only if

$$
\widehat{\Upsilon}(i \xi, i \eta)=e^{-\frac{1}{2}(\langle\xi, \xi\rangle+\langle\eta, \eta\rangle)-2\langle\xi, \eta\rangle},
$$

which is equivalent to $\Upsilon=e^{\frac{1}{2} \Delta_{G}^{*}} \Gamma(2 I) e^{\frac{1}{2} \Delta_{\mathrm{G}}}$.

Proposition 3.2. Let $\Upsilon \in \mathcal{L}\left((E),(E)^{*}\right)$ be given. If $\Upsilon=\Gamma(I)$, then for any $\Xi_{1}, \Xi_{2} \in \mathcal{L}\left((E),(E)^{*}\right)$, the convolution $\Xi_{1} *_{\Upsilon} \Xi_{2}$ coincides with the convolution $\Xi_{1} * \Xi_{2}$ in [1]. In this case, for $\xi, \eta \in E, T_{\mathrm{op}}\left(\Xi_{1} * \Xi_{2}\right)(\xi, \eta)$ is given by

$$
T_{\mathrm{op}}\left(\Xi_{1} * \Xi_{2}\right)(\xi, \eta)=T_{\mathrm{op}}\left(\Xi_{1}\right)(\xi, \eta) T_{\mathrm{op}}\left(\Xi_{2}\right)(\xi, \eta) e^{\frac{1}{2}\langle\xi+\eta, \xi+\eta\rangle} .
$$

Proof. The proof is straightforward from (4).

\subsection{Relations between convolutions and transforms}

By applying Theorem 2.1, we can easily see that there exists an operator $\mathcal{G}_{\alpha, \beta} \in \mathcal{L}((E),(E))$ such that

$$
\mathcal{G}_{\alpha, \beta} \phi_{\xi}=\phi_{\beta \xi} \exp \{\alpha\langle\xi, \xi\rangle\}, \quad \xi \in E .
$$

From (5) and (2), we can easily see that

$$
\mathcal{G}_{\alpha, \beta}=\Gamma(\beta I) e^{\alpha \Delta_{\mathrm{G}}},
$$


which is called the Fourier-Gauss transform. The adjoint operator of $\mathcal{G}_{\alpha, \beta}$ is denoted by $\mathcal{F}_{\alpha, \beta} \in \mathcal{L}\left((E)^{*},(E)^{*}\right)$ and called the generalized Fourier-Mehler transform. Then by the duality of (6), we have

$$
\mathcal{F}_{\alpha, \beta}=e^{\alpha \Delta_{G}^{*}} \Gamma(\beta I)
$$

If $\alpha(\theta)=\frac{1}{2} i e^{i \theta} \sin \theta$ and $\beta(\theta)=e^{i \theta}$ for $\theta \in \mathbb{R}$, then $\mathcal{F}_{\theta} \equiv \mathcal{F}_{\alpha(\theta), \beta(\theta)}$ is called the Fourier-Mehler transform. For more details, we refer to [3].

Let $\alpha, \beta, \gamma, \delta \in \mathbb{C}$. The quantum generalized Fourier-Mehler transform $\mathcal{F}_{\alpha, \beta ; \gamma, \delta}^{Q} \in \mathcal{L}\left(\mathcal{L}\left((E),(E)^{*}\right)\right)$ studied in [7] is defined by

$$
\mathcal{F}_{\alpha, \beta ; \gamma, \delta}^{Q}(\Xi)=\mathcal{F}_{\alpha, \gamma} \Xi \mathcal{G}_{\beta, \delta}, \quad \Xi \in \mathcal{L}\left((E),(E)^{*}\right)
$$

Theorem 3.3. Let $\alpha_{i}, \beta_{i} \in \mathbb{C}$ and $\gamma_{i}, \delta_{i} \in \mathbb{C}^{*}=\mathbb{C} \backslash\{0\}$ for $i=1,2,3$, and $\Upsilon, \Upsilon^{\prime} \in \mathcal{L}\left((E),(E)^{*}\right)$ be given. Then for any $\Xi_{1}, \Xi_{2} \in \mathcal{L}\left((E),(E)^{*}\right)$,

$$
\mathcal{F}_{\alpha_{1}, \beta_{1} ; \gamma_{1}, \delta_{1}}^{Q}\left(\Xi_{1} * \Upsilon \Xi_{2}\right)=\mathcal{F}_{\alpha_{2}, \beta_{2} ; \gamma_{2}, \delta_{2}}^{Q}\left(\Xi_{1}\right) * \Upsilon^{\prime} \mathcal{F}_{\alpha_{3}, \beta_{3} ; \gamma_{3}, \delta_{3}}^{Q}\left(\Xi_{2}\right)
$$

if and only if $\gamma_{1}=\gamma_{2}=\gamma_{3}, \delta_{1}=\delta_{2}=\delta_{3}$ and

$$
\begin{aligned}
\Upsilon= & {\left[\Gamma\left(\gamma_{1}^{-1} I\right) \Upsilon^{\prime} \Gamma\left(\delta_{1}^{-1} I\right)\right] } \\
& \times \diamond\left[e^{\left(\alpha_{2}+\alpha_{3}-\alpha_{1}\right) \gamma_{1}^{-2} \Delta_{G}^{*}} \Gamma\left(\left(3-2\left(\gamma_{1} \delta_{1}\right)^{-1}\right) I\right) e^{\left(\beta_{2}+\beta_{3}-\beta_{1}\right) \delta_{1}^{-2} \Delta_{\mathrm{G}}}\right] .
\end{aligned}
$$

Proof. By (7) and (1) we obtain that for any $\xi, \eta \in E$,

$$
\begin{aligned}
& \left(\mathcal{F}_{\alpha_{1}, \beta_{1} ; \gamma_{1}, \delta_{1}}^{Q}\left(\Xi_{1} * \Upsilon \Xi_{2}\right)\right)(\xi, \eta) \\
= & \left\langle\left\langle\left(\Xi_{1} \diamond \Xi_{2} \diamond \Upsilon\right) \phi_{\delta_{1} \xi}, \phi_{\gamma_{1} \eta}\right\rangle\right\rangle e^{\beta_{1}\langle\xi, \xi\rangle+\alpha_{1}\langle\eta, \eta\rangle} \\
= & \left\langle\left\langle\Xi_{1} \phi_{\delta_{1} \xi}, \phi_{\gamma_{1} \eta}\right\rangle\right\rangle\left\langle\left\langle\Xi_{2} \phi_{\delta_{1} \xi}, \phi_{\gamma_{1} \eta}\right\rangle\right\rangle\left\langle\left\langle\Upsilon \phi_{\delta_{1} \xi}, \phi_{\gamma_{1} \eta}\right\rangle\right\rangle \\
& \times e^{\beta_{1}\langle\xi, \xi\rangle+\alpha_{1}\langle\eta, \eta\rangle-2\left\langle\delta_{1} \xi, \gamma_{1} \eta\right\rangle},
\end{aligned}
$$

and

$$
\begin{aligned}
& \left(\mathcal{F}_{\alpha_{2}, \beta_{2} ; \gamma_{2}, \delta_{2}}^{Q}\left(\Xi_{1}\right) \diamond \mathcal{F}_{\alpha_{3}, \beta_{3} ; \gamma_{3}, \delta_{3}}^{Q}\left(\Xi_{2}\right) \diamond \Upsilon^{\prime}\right)^{-}(\xi, \eta) \\
= & \left\langle\left\langle\Xi_{1} \phi_{\delta_{2} \xi}, \phi_{\gamma_{2} \eta}\right\rangle\right\rangle\left\langle\left\langle\Xi_{2} \phi_{\delta_{3} \xi}, \phi_{\gamma_{3} \eta}\right\rangle\right\rangle \widehat{\Upsilon}^{\prime}(\xi, \eta) \\
& \times e^{\left(\beta_{2}+\beta_{3}\right)\langle\xi, \xi\rangle+\left(\alpha_{2}+\alpha_{3}\right)\langle\eta, \eta\rangle-2\langle\xi, \eta\rangle}
\end{aligned}
$$

By comparing (10) and (11), we see that (8) holds if and only if $\gamma_{1}=\gamma_{2}=\gamma_{3}$, $\delta_{1}=\delta_{2}=\delta_{3}$ and for any $\xi, \eta \in E$,

(12) $\widehat{\Upsilon}\left(\delta_{1} \xi, \gamma_{1} \eta\right)=\widehat{\Upsilon^{\prime}}(\xi, \eta) e^{\left(\alpha_{2}+\alpha_{3}-\alpha_{1}\right)\langle\eta, \eta\rangle+2\left(\gamma_{1} \delta_{1}-1\right)\langle\xi, \eta\rangle+\left(\beta_{2}+\beta_{3}-\beta_{1}\right)\langle\xi, \xi\rangle}$,

which is equivalent to $(9)$.

Corollary 3.4. Let $\alpha_{i}, \beta_{i} \in \mathbb{C}$ for $i=1,2,3, \gamma, \delta \in \mathbb{C}^{*}$ and $\Upsilon \in \mathcal{L}\left((E),(E)^{*}\right)$ be given. Then for any $\Xi_{1}, \Xi_{2} \in \mathcal{L}\left((E),(E)^{*}\right)$,

$$
\mathcal{F}_{\alpha_{1}, \beta_{1} ; \gamma, \delta}^{Q}\left(\Xi_{1} * \Upsilon \Xi_{2}\right)=\mathcal{F}_{\alpha_{2}, \beta_{2} ; \gamma, \delta}^{Q}\left(\Xi_{1}\right) \diamond \mathcal{F}_{\alpha_{3}, \beta_{3} ; \gamma, \delta}^{Q}\left(\Xi_{2}\right)
$$


if and only if

$$
\Upsilon=e^{\left(\alpha_{2}+\alpha_{3}-\alpha_{1}\right) \gamma^{-2} \Delta_{G}^{*}} \Gamma\left(\left(2-(\gamma \delta)^{-1}\right) I\right) e^{\left(\beta_{2}+\beta_{3}-\beta_{1}\right) \delta^{-2} \Delta_{\mathrm{G}}} .
$$

Proof. Since $\Gamma(I)$ is the identity with respect to the Wick product, then ${ }^{*} \Gamma(I)$ coincides with the Wick product $\diamond$. Therefore, by applying Theorem 3.3, the proof is straightforward.

The following corollary is immediate from Corollary 3.4.

Corollary 3.5. Let $\alpha, \beta \in \mathbb{C}$ and $\gamma, \delta \in \mathbb{C}^{*}$ and $\Xi_{1}, \Xi_{2} \in \mathcal{L}\left((E),(E)^{*}\right)$. Then

$$
\mathcal{F}_{\alpha, \beta ; \gamma, \delta}^{Q}\left(\Xi_{1} * \Upsilon \Xi_{2}\right)=\mathcal{F}_{\alpha, \beta ; \gamma, \delta}^{Q}\left(\Xi_{1}\right) \diamond \mathcal{F}_{\alpha, \beta ; \gamma, \delta}^{Q}\left(\Xi_{2}\right)
$$

if and only if $\Upsilon=e^{\alpha \gamma^{-2} \Delta_{G}^{*}} \Gamma\left(\left(2-(\gamma \delta)^{-1}\right) I\right) e^{\beta \delta^{-2} \Delta_{\mathrm{G}}}$.

Corollary 3.6. Let $\alpha_{i}, \beta_{i} \in \mathbb{C}$ for $i=1,2,3, \gamma, \delta \in \mathbb{C}^{*}$ and $\Upsilon \in \mathcal{L}\left((E),(E)^{*}\right)$ be given. Then for any $\Xi_{1}, \Xi_{2} \in \mathcal{L}\left((E),(E)^{*}\right)$,

$$
\mathcal{F}_{\alpha_{1}, \beta_{1} ; \gamma, \delta}^{Q}\left(\Xi_{1} \diamond \Xi_{2}\right)=\mathcal{F}_{\alpha_{2}, \beta_{2} ; \gamma, \delta}^{Q}\left(\Xi_{1}\right) * \Upsilon \mathcal{F}_{\alpha_{3}, \beta_{3} ; \gamma, \delta}^{Q}\left(\Xi_{2}\right)
$$

if and only if

$$
\Upsilon=e^{\alpha \Delta_{G}^{*}} \Gamma((2-\gamma \delta) I) e^{\beta \Delta_{\mathrm{G}}}
$$

with $\alpha=-\left(\alpha_{2}+\alpha_{3}-\alpha_{1}\right)$ and $\beta=-\left(\beta_{2}+\beta_{3}-\beta_{1}\right)$.

Proof. The proof is a simple application of Theorem 3.3 with the fact that $\left(e^{\diamond \Xi}\right)^{\diamond(-1)}=e^{\diamond(-\Xi)}$, whenever the Wick exponential:

$$
e^{\diamond \Xi}=\sum_{n=0}^{\infty} \frac{1}{n !} \Xi^{\diamond n}
$$

is well defined as a white noise operator in $\mathcal{L}\left((E),(E)^{*}\right)$. Here $\Xi^{\diamond(-1)}$ stands the (Wick) inverse operator of $\Xi \in \mathcal{L}\left((E),(E)^{*}\right)$ with respect to the Wick product. Also, we can easily check that $\Gamma(\rho I)^{\diamond(-1)}=\Gamma((2-\rho) I)$ for any $\rho \in \mathbb{C}$. Since we know that

$$
e^{\varrho \Delta_{G}^{*}} \Gamma(\rho I) e^{\sigma \Delta_{\mathrm{G}}}=e^{\varrho \Delta_{G}^{*}} \diamond \Gamma(\rho I) \diamond e^{\sigma \Delta_{\mathrm{G}}}
$$

for any $\rho, \varrho, \sigma \in \mathbb{C}$, from (9) we have

$$
\Upsilon=\Gamma(\gamma I)\left[e^{\alpha \gamma^{-2} \Delta_{G}^{*}} \Gamma\left(\left(2(\gamma \delta)^{-1}-1\right) I\right) e^{\beta \delta^{-2} \Delta_{\mathrm{G}}}\right] \Gamma(\delta I)
$$

with $\alpha=-\left(\alpha_{2}+\alpha_{3}-\alpha_{1}\right)$ and $\beta=-\left(\beta_{2}+\beta_{3}-\beta_{1}\right)$. On the other hand, for any $\rho, \sigma \in \mathbb{C}$, we can easily check that

$$
e^{\sigma \Delta_{\mathrm{G}}} \Gamma(\rho I)=\Gamma(\rho I) e^{\sigma \rho^{2} \Delta_{\mathrm{G}}}
$$

and by duality,

$$
\Gamma(\rho I) e^{\sigma \Delta_{\mathrm{G}}^{*}}=e^{\sigma \rho^{2} \Delta_{\mathrm{G}}^{*}} \Gamma(\rho I) .
$$

Therefore, from (14) we see that $\Upsilon$ is of the form as in (13). 
Remark 3.7. By applying (12) we can prove Corollary 3.6 directly. However, in the proof of Corollary 3.6 we used the Wick calculus of white noise operators which is a useful tool to study equations associated with Wick product arising in the quantum field theory.

The following corollary is immediate from Corollary 3.6.

Corollary 3.8. Let $\alpha, \beta \in \mathbb{C}, \gamma, \delta \in \mathbb{C}^{*}$ and $\Upsilon \in \mathcal{L}\left((E),(E)^{*}\right)$ be given. Then for any $\Xi_{1}, \Xi_{2} \in \mathcal{L}\left((E),(E)^{*}\right)$,

if and only if

$$
\mathcal{F}_{\alpha, \beta ; \gamma, \delta}^{Q}\left(\Xi_{1} \diamond \Xi_{2}\right)=\mathcal{F}_{\alpha, \beta ; \gamma, \delta}^{Q}\left(\Xi_{1}\right) * \Upsilon \mathcal{F}_{\alpha, \beta ; \gamma, \delta}^{Q}\left(\Xi_{2}\right)
$$

$$
\Upsilon=e^{-\alpha \Delta_{G}^{*}} \Gamma((2-\gamma \delta) I) e^{-\beta \Delta_{\mathrm{G}}}
$$

\section{Quantum-classical correspondence}

\section{1. $S$-transform, $T$-transform and convolution}

The $S$-transform of an element $\Phi \in(E)^{*}$ is a complex valued function on $E$ defined by

$$
S \Phi(\xi)=\left\langle\left\langle\Phi, \phi_{\xi}\right\rangle, \quad \xi \in E .\right.
$$

Then every $\Phi \in(E)^{*}$ is uniquely determined by its $S$-transform since $\left\{\phi_{\xi}: \xi \in E\right\}$ spans a dense subspace of $(E)$. The Wick product $\Phi \diamond \Psi$ of $\Phi$ and $\Psi$ in $(E)^{*}$ is defined as the unique element of $(E)^{*}$ such that $S(\Phi \diamond \Psi)=S(\Phi) S(\Psi)$. Then $\left((E)^{*}, \diamond\right)$ is a commutative algebra. The T-transform $T \Phi$ of $\Phi \in(E)^{*}$ is defined to be a complex valued function on $E$ satisfying

$$
T \Phi(\xi)=e^{-\frac{1}{2}\langle\xi, \xi\rangle} S \Phi(i \xi), \quad \xi \in E,
$$

see $[5,11]$. For each $\Xi \in \mathcal{L}\left((E),(E)^{*}\right)$ and any $\xi \in E$, we have

$$
T_{\mathrm{OP}}(\Xi)(0, \xi)=\widehat{\Xi}(0, i \xi) e^{-\frac{1}{2}\langle\xi, \xi\rangle}=T\left(\Xi \phi_{0}\right)(\xi),
$$

where $\phi_{0}=(1,0, \ldots) \in(E)$ is the vacuum vector.

For fixed $\mathbf{F} \in(E)^{*}$, a convolution $\Phi *_{\mathbf{F}} \Psi$ of $\Phi, \Psi \in(E)^{*}$ is defined by

$$
\Phi *_{\mathbf{F}} \Psi=\Phi \diamond \Psi \diamond \mathbf{F} .
$$

In particular, the convolution product $*_{\mathbf{F}}$ with $\mathbf{F}=g_{c}$ coincides with the convolution product $*$ studied in [11], i.e., for any $\Phi, \Psi \in(E)^{*}$

$$
\Phi *_{g_{-2}} \Psi=\Phi * \Psi \equiv \Phi \diamond \Psi \diamond g_{-2},
$$

where $g_{c} \in(E)$ is the Gaussian (white noise) function with mean 0 and variance $c$ such that

$$
S g_{c}(\xi)=\exp \left\{-\frac{1}{2(1+c)}\langle\xi, \xi\rangle\right\}, \quad c \neq-1 .
$$

Lemma 4.1. For each $c \in \mathbb{C}$ with $c \neq 0$ we have

$$
e^{c \Delta_{\mathrm{G}}^{*}} \phi_{0}=g_{-\left(1+\frac{1}{2 c}\right)} \text {. }
$$


Proof. For any $\xi \in E$, we have

$$
S\left(e^{c \Delta_{\mathrm{G}}^{*}} \phi_{0}\right)(\xi)=\left\langle\left\langle\phi_{0}, e^{c \Delta_{\mathrm{G}}} \phi_{\xi}\right\rangle\right\rangle=e^{c\langle\xi, \xi\rangle}
$$

and so the proof is immediate from (16).

\subsection{Quantum-classical correspondence}

For $\Phi \in(E)^{*}$ and $\phi \in(E)$, the (pointwise) multiplication $\Phi \phi \in(E)^{*}$ is well-defined as

$$
\langle\langle\Phi \phi, \psi\rangle\rangle=\langle\langle\Phi, \phi \psi\rangle\rangle, \quad \psi \in(E) .
$$

Here we use the fact that $(E)$ is closed under the (pointwise) multiplication as white noise functionals (Cauchy product of vectors), see [13]. For each $\Phi \in(E)^{*}$, let $M_{\Phi} \in \mathcal{L}\left((E),(E)^{*}\right)$ be the multiplication operator associated with $\Phi$, i.e., $M_{\Phi}(\phi)=\Phi \phi$ for any $\phi \in(E)$.

For each $\Xi_{1}, \Xi_{2} \in \mathcal{L}\left((E),(E)^{*}\right)$ we can easily see that

$$
\left(\Xi_{1} \diamond \Xi_{2}\right) \phi_{0}=\left(\Xi_{1} \phi_{0}\right) \diamond\left(\Xi_{2} \phi_{0}\right)
$$

and so for each $\Phi, \Psi \in(E)^{*},\left(M_{\Phi} \diamond M_{\Psi}\right) \phi_{0}=\Phi \diamond \Psi$, more generally, we have the following lemma.

Lemma 4.2. Let $\mathbf{F} \in(E)^{*}$. Then for any $\Phi, \Psi \in(E)^{*}$,

$$
M_{\Phi} \diamond M_{\Psi}=M_{\Phi \diamond \Psi}, \quad M_{\Phi} *_{M_{\mathbf{F}}} M_{\Psi}=M_{\Phi *_{\mathrm{F}} \Psi}
$$

Proof. The second identity is immediate from the definition and the first identity. The proof of the first identity is straightforward by taking operator symbols on both sides using the fact that $\phi_{\xi} \phi_{\eta}=\phi_{\xi+\eta} e^{\langle\xi, \eta\rangle}$ for any $\xi, \eta \in E$.

By applying Proposition 3.1 with (15) and (18) we have the following proposition.

Proposition 4.3. Let $\mathbf{F} \in(E)^{*}$ be given. Then for any $\Phi, \Psi \in(E)^{*}$,

$$
T\left(\Phi *_{\mathbf{F}} \Psi\right)=T(\Phi) T(\Psi)
$$

if and only if $\mathbf{F}=g_{-2}$.

Proof. By applying Proposition 3.1 with (15) and (18) we can see that (19) holds if and only if $\mathbf{F}=\Upsilon \phi_{0}$ with $\Upsilon=e^{\frac{1}{2} \Delta_{G}^{*}} \Gamma(2 I) e^{\frac{1}{2} \Delta_{\mathrm{G}}}$. On the other hand, by Lemma 4.1 we have $\Upsilon \phi_{0}=e^{\frac{1}{2} \Delta_{G}^{*}} \phi_{0}=g_{-2}$.

Theorem 4.4. Let $\alpha_{i} \in \mathbb{C}$ for $i=1,2,3$ and $\gamma \in \mathbb{C}^{*}$. Let $\mathbf{F}, \mathbf{G} \in(E)^{*}$ be given. Then for any $\Phi, \Psi \in(E)^{*}$,

$$
\mathcal{F}_{\alpha_{1}, \gamma}\left(\Phi *_{\mathbf{F}} \Psi\right)=\mathcal{F}_{\alpha_{2}, \gamma}(\Phi) *_{\mathbf{G}} \mathcal{F}_{\alpha_{3}, \gamma}(\Psi)
$$

if and only if

$$
\mathbf{F}=e^{\left(\alpha_{2}+\alpha_{3}-\alpha_{1}\right) \gamma^{-2} \Delta_{\mathrm{G}}^{*}} \Gamma\left(\gamma^{-1} I\right) \mathbf{G} .
$$


Proof. For any $\xi \in E$, we obtain that

$$
S\left(\mathcal{F}_{\alpha_{1}, \gamma}\left(\Phi *_{\mathbf{F}} \Psi\right)\right)(\xi)=e^{\alpha_{1}\langle\xi, \xi\rangle} S \Phi(\gamma \xi) S \Psi(\gamma \xi) S \mathbf{F}(\gamma \xi)
$$

and

$$
S\left(\mathcal{F}_{\alpha_{2}, \gamma}(\Phi) *_{\mathbf{G}} \mathcal{F}_{\alpha_{3}, \gamma}(\Psi)\right)(\xi)=e^{\left(\alpha_{2}+\alpha_{3}\right)\langle\xi, \xi\rangle} S \Phi(\gamma \xi) S \Psi(\gamma \xi) S \mathbf{G}(\xi) .
$$

Therefore, (20) holds if and only if for any $\xi \in E$

$$
S \mathbf{F}(\gamma \xi)=e^{\left(\alpha_{2}+\alpha_{3}-\alpha_{1}\right)\langle\xi, \xi\rangle} S \mathbf{G}(\xi),
$$

which is equivalent to (21).

Remark 4.5. In Theorem 4.4, if $\alpha_{2}+\alpha_{3} \neq \alpha_{1}$, then by taking $S$-transform on (21), we can easily see that

$$
\mathbf{F}=\Gamma\left(\gamma^{-1} I\right) \mathbf{G} \diamond g_{d}, \quad d=-\left(1+\frac{1}{2\left(\alpha_{2}+\alpha_{3}-\alpha_{1}\right) \gamma^{-2}}\right) .
$$

Lemma 4.6. Let $\mathbf{G} \in(E)^{*}$ and $\alpha, \gamma \in \mathbb{C}$. Then we have

$$
M_{e^{\alpha \Delta_{\mathrm{G}}^{*}}(\gamma I) \mathbf{G}}=\left[\Gamma(\gamma I) M_{\mathbf{G}} \Gamma(\gamma I)\right] \diamond e^{\alpha \Delta_{\mathrm{G}}^{*}} \Gamma\left(\left(2 \alpha+2-\gamma^{2}\right) I\right) e^{\alpha \Delta_{\mathrm{G}}} .
$$

Proof. For any $\xi, \eta \in E$ we have

$$
\begin{aligned}
M_{e^{\alpha \Delta_{\mathrm{G}}^{*} \Gamma(\gamma I) \mathbf{G}}}(\xi, \eta)= & \left\langle\left\langle e^{\alpha \Delta_{\mathrm{G}}^{*}} \Gamma(\gamma I) \mathbf{G}, \phi_{\xi+\eta}\right\rangle\right\rangle e^{\langle\xi, \eta\rangle} \\
= & \left\langle\left\langle\mathbf{G}, \phi_{\gamma(\xi+\eta)}\right\rangle\right\rangle e^{\alpha\langle\xi+\eta, \xi+\eta\rangle+\langle\xi, \eta\rangle} \\
= & \left\langle\left\langle\mathbf{G}, \phi_{\gamma \xi} \phi_{\gamma \eta}\right\rangle e^{\alpha\langle\xi, \xi\rangle+\alpha\langle\eta, \eta\rangle+\left(2 \alpha+2-\gamma^{2}\right)\langle\xi, \eta\rangle-\langle\xi, \eta\rangle}\right. \\
= & \left\langle\left\langle\Gamma(\gamma I) M_{\mathbf{G}} \Gamma(\gamma I) \phi_{\xi}, \phi_{\eta}\right\rangle\right\rangle \\
& \quad \times e^{\alpha\langle\xi, \xi\rangle+\alpha\langle\eta, \eta\rangle+\left(2 \alpha+2-\gamma^{2}\right)\langle\xi, \eta\rangle-\langle\xi, \eta\rangle}
\end{aligned}
$$

where we used the fact that $\phi_{\xi} \phi_{\eta}=\phi_{\xi+\eta} e^{\langle\xi, \eta\rangle}$, which implies (22).

For any $\alpha, \beta, \gamma, \delta \in \mathbb{C}$ and $\Xi \in \mathcal{L}\left((E),(E)^{*}\right)$ we can easily check that

$$
\mathcal{F}_{\alpha, \beta ; \gamma, \delta}^{Q}(\Xi) \phi_{0}=\mathcal{F}_{\alpha, \gamma}\left(\Xi \phi_{0}\right)
$$

and so, by applying (17), for any $\mathbf{F} \in(E)^{*}$ we have

$$
\mathcal{F}_{\alpha, \beta ; \gamma, \delta}^{Q}\left(\Xi_{1} *_{M_{\mathbf{F}}} \Xi_{2}\right) \phi_{0}=\mathcal{F}_{\alpha, \gamma}\left(\Xi_{1} \phi_{0} *_{\mathbf{F}} \Xi_{2} \phi_{0}\right)
$$

for any $\Xi_{1}, \Xi_{2} \in \mathcal{L}\left((E),(E)^{*}\right)$.

Theorem 4.7. Let $\alpha_{i} \in \mathbb{C}$ for $i=1,2,3$ and $\gamma \in \mathbb{C}^{*}$ with $\alpha_{2}+\alpha_{3}-\alpha_{1}=\frac{\gamma^{2}-1}{2}$. Let $\mathbf{F}, \mathbf{G} \in(E)^{*}$ be given. Then for any $\Xi_{1}, \Xi_{2} \in \mathcal{L}\left((E),(E)^{*}\right)$,

$$
\mathcal{F}_{\alpha_{1}, \alpha_{1} ; \gamma, \gamma}\left(\Xi_{1} *_{M_{\mathbf{F}}} \Xi_{2}\right)=\mathcal{F}_{\alpha_{2}, \alpha_{2} ; \gamma, \gamma}\left(\Xi_{1}\right) *_{M_{\mathbf{G}}} \mathcal{F}_{\alpha_{3}, \alpha_{3} ; \gamma, \gamma}\left(\Xi_{2}\right)
$$

if and only if

$$
\mathbf{F}=e^{\frac{1}{2}\left(1-\gamma^{-2}\right) \Delta_{\mathrm{G}}^{*}} \Gamma\left(\gamma^{-1} I\right) \mathbf{G} .
$$


Proof. $\Rightarrow)$ By acting vacuum vector $\phi_{0}$ on the both sides of $(23)$, we have

$$
\mathcal{F}_{\alpha_{1}, \gamma}\left(\Xi_{1} \phi_{0} *_{\mathbf{F}} \Xi_{2} \phi_{0}\right)=\mathcal{F}_{\alpha_{2}, \gamma}\left(\Xi_{1} \phi_{0}\right) *_{\mathbf{G}} \mathcal{F}_{\alpha_{3}, \gamma}\left(\Xi_{2} \phi_{0}\right) \text {. }
$$

Therefore, by Theorem 4.4, (24) holds.

$\Leftarrow)$ Suppose that $(24)$ holds. Then by Lemma 4.6, we have

$$
M_{\mathbf{F}}=\Gamma\left(\gamma^{-1} I\right) M_{\mathbf{G}} \Gamma\left(\gamma^{-1} I\right) \diamond e^{c \Delta_{\mathrm{G}}^{*}} \Gamma\left(\left(3-2 \gamma^{-2}\right) I\right) e^{c \Delta_{\mathrm{G}}}
$$

with $c=\left(\alpha_{2}+\alpha_{3}-\alpha_{1}\right) \gamma^{-2}$ and $\alpha_{2}+\alpha_{3}-\alpha_{1}=\frac{\gamma^{2}-1}{2}$. Therefore, by Theorem 3.3 , (23) holds.

Corollary 4.8. Let $\alpha \in \mathbb{C}$ and $\gamma \in \mathbb{C}^{*}$. Let $\mathbf{F}, \mathbf{G} \in(E)^{*}$ be given. Then for any $\Phi, \Psi \in(E)^{*}$,

$$
\mathcal{F}_{\alpha, \gamma}\left(\Phi *_{\mathbf{F}} \Psi\right)=\mathcal{F}_{\alpha, \gamma}(\Phi) *_{\mathbf{G}} \mathcal{F}_{\alpha, \gamma}(\Psi)
$$

if and only if

$$
\mathbf{F}=e^{\alpha \gamma^{-2} \Delta_{\mathrm{G}}^{*}} \Gamma\left(\gamma^{-1} I\right) \mathbf{G} .
$$

In particular, by (26) and Lemma 4.1, for any $\Phi, \Psi \in(E)^{*}$,

$$
\mathcal{F}_{\alpha, \gamma}\left(\Phi *_{-\left(1+\frac{\gamma^{2}}{2 \alpha}\right)} \Psi\right)=\mathcal{F}_{\alpha, \gamma}(\Phi) \diamond \mathcal{F}_{\alpha, \gamma}(\Psi)
$$

for $\alpha \neq 0$, which is a special case of (26) with $\mathbf{G}=\phi_{0}$.

Proof. The proof is immediate from Theorem 4.4.

Let $\mathbf{F}, \mathbf{G} \in(E)^{*}$ be given such that $(26)$ holds. Then we have

$$
\mathbf{G}=\Gamma(\gamma I) e^{-\alpha \gamma^{-2} \Delta_{\mathrm{G}}^{*}} \mathbf{F} .
$$

On the other hand,

$$
\Gamma(\gamma I) e^{-\alpha \gamma^{-2} \Delta_{\mathrm{G}}^{*}}=e^{-\alpha \Delta_{\mathrm{G}}^{*}} \Gamma(\gamma I)
$$

and so

$$
\mathbf{G}=e^{-\alpha \Delta_{\mathrm{G}}^{*}} \Gamma(\gamma I) \mathbf{F}=\Gamma(\gamma I) \mathbf{F} \diamond g_{\frac{1}{2 \alpha}-1},
$$

for the second equality we assume that $\alpha \neq 0$. If $\mathbf{F}=\phi_{0}$, then for $\alpha \neq 0$ by Lemma 4.1 we have

$$
\mathbf{G}=e^{-\alpha \Delta_{\mathrm{G}}^{*}} \Gamma(\gamma I) \phi_{0}=e^{-\alpha \Delta_{\mathrm{G}}^{*}} \phi_{0}=g_{\frac{1}{2 \alpha}-1} .
$$

Therefore, by (25), we have the following corollary.

Corollary 4.9. Let $\alpha, \gamma \in \mathbb{C}^{*}$. Then for any $\Phi, \Psi \in(E)^{*}$,

$$
\mathcal{F}_{\alpha, \gamma}(\Phi \diamond \Psi)=\mathcal{F}_{\alpha, \gamma}(\Phi) *_{g_{\frac{1}{2 \alpha}-1}} \mathcal{F}_{\alpha, \gamma}(\Psi)
$$

in particular,

$$
\mathcal{F}_{\theta}(\Phi \diamond \Psi)=\mathcal{F}_{\theta}(\Phi) *_{g_{-(2+i \cot \theta)}} \mathcal{F}_{\theta}(\Psi)
$$

for any $\theta \in \mathbb{R}$, see [11]. 
Let $\mathbf{F}, \mathbf{G} \in(E)^{*}$ be given such that $(26)$ holds. If $\mathbf{F}=g_{c}$ for $c \neq-1$ and $\gamma^{2} \neq-2 \alpha(1+c) \in \mathbb{C}^{*}$, then from $(27)$ we have

$$
\mathbf{G}_{c}=\Gamma(\gamma I) g_{c} \diamond g_{\frac{1}{2 \alpha}-1}=g_{d}, \quad d=\frac{1+c}{\gamma^{2}+2 \alpha(1+c)}-1 .
$$

In particular, $\mathbf{G}_{-2}=g_{-\left(1+\frac{1}{\gamma^{2}-2 \alpha}\right)}$ for $\gamma^{2} \neq 2 \alpha \in \mathbb{C}^{*}$. Therefore, the following corollary is immediate from Corollary 4.8.

Corollary 4.10. Let $\alpha, \gamma \in \mathbb{C}^{*}$ and $c \in \mathbb{C}$ with $\alpha \neq-1$ and $\gamma^{2} \neq-2 \alpha(1+c)$. Then for any $\Phi, \Psi \in(E)^{*}$,

$$
\mathcal{F}_{\alpha, \gamma}\left(\Phi *_{g_{c}} \Psi\right)=\mathcal{F}_{\alpha, \gamma}(\Phi) *_{\mathbf{G}_{c}} \mathcal{F}_{\alpha, \gamma}(\Psi),
$$

where $\mathbf{G}_{c}$ is given as in (28). In particular, for any $\Phi, \Psi \in(E)^{*}$,

$$
\mathcal{F}_{\alpha, \gamma}(\Phi * \Psi)=\mathcal{F}_{\alpha, \gamma}(\Phi) *_{\mathbf{G}_{-2}} \mathcal{F}_{\alpha, \gamma}(\Psi)
$$

for $\gamma^{2} \neq 2 \alpha$, specially,

$$
\mathcal{F}_{\theta}(\Phi * \Psi)=\mathcal{F}_{\theta}(\Phi) *_{g_{i \tan \theta-2}} \mathcal{F}_{\theta}(\Psi)
$$

for any $\theta \in \mathbb{R}$, see [11].

\section{References}

[1] M. Ben Chrouda, M. El Ouled, and H. Ouerdiane, Quantum stochastic processes and applications, Quantum probability and infinite dimensional analysis, 115-125, QP-PQ: Quantum Probab. White Noise Anal., 18, World Sci. Publ., Hackensack, NJ, 2005.

[2] D. M. Chung and U. C. Ji, Transforms on white noise functionals with their applications to Cauchy problems, Nagoya Math. J. 147 (1997), 1-23.

[3] - Transformation groups on white noise functionals and their applications, Appl. Math. Optim. 37 (1998), no. 2, 205-223.

[4] D. M. Chung, U. C. Ji, and N. Obata, Quantum stochastic analysis via white noise operators in weighted Fock space, Rev. Math. Phys. 14 (2002), no. 3, 241-272.

[5] T. Hida, Analysis of Brownian Functionals, Carleton Math. Lect. Notes no. 13, Carleton University, Ottawa, 1975.

[6] R. L. Hudson and K. R. Parthasarathy, Quantum Ito's formula and stochastic evolutions, Comm. Math. Phys. 93 (1984), no. 3, 301-323.

[7] U. C. Ji, Quantum extensions of Fourier-Gauss and Fourier-Mehler transforms, J. Korean Math. Soc. 45 (2008), no. 6, 1785-1801.

[8] U. C. Ji and N. Obata, Quantum white noise calculus, in "Non-Commutativity, InfiniteDimensionality and Probability at the Crossroads (N. Obata, T. Matsui and A. Hora, Eds.)," pp. 143-191, World Scientific, 2002.

[9] _ A unified characterization theorem in white noise theory, Infin. Dimens. Anal. Quantum Probab. Relat. Top. 6 (2003), no. 2, 167-178.

[10] _ Annihilation-derivative, creation-derivative and representation of quantum martingales, Comm. Math. Phys. 286 (2009), no. 2, 751-775.

[11] H.-H. Kuo, White Noise Distribution Theory, CRC Press, 1996.

[12] P.-A. Meyer, Quantum Probability for Probabilists, Lect. Notes in Math. Vol. 1538, Springer-Verlag, 1993.

[13] N. Obata, White Noise Calculus and Fock Space, Lect. Notes in Math. Vol. 1577, Springer-Verlag, 1994.

[14] _ An analytic characterization of symbols of operators on white noise functionals, J. Math. Soc. Japan 45 (1993), no. 3, 421-445. 
[15] K. R. Parthasarathy, An Introduction to Quantum Stochastic Calculus, Birkhäuser, 1992.

UN Cig Ji

Department of Mathematics

Research Institute of Mathematical Finance

Chungbuk National University

Cheonguu 361-763, Korea

E-mail address: uncigji@chungbuk.ac.kr

Young Yi KIM

Department of Mathematics

Chungbuk National University

Cheongue 361-763, Korea

E-mail address: kimyy@chungbuk.ac.kr 